\title{
Gene expression-based immune infiltration analyses of renal cancer and their associations with survival outcome
}

\section{Lei Chen}

Cangzhou Central Hospital

Liang Yin

Cangzhou Central Hospital

\section{Zilong Qi}

Cangzhou Central Hospital

Jinmin Li

Cangzhou Central Hospital

Xinning Wang

Cangzhou Central Hospital

Kun Ma

Cangzhou Central Hospital

Xiangyang Liu ( $\square$ liuxiangyangcz@outlook.com )

Cangzhou Central Hospital https://orcid.org/0000-0002-8589-2989

\section{Research article}

Keywords: renal cancer, tumor-infiltrating immune cells, proportion, prognosis

Posted Date: January 5th, 2021

DOl: https://doi.org/10.21203/rs.3.rs-19552/v3

License: (9) (i) This work is licensed under a Creative Commons Attribution 4.0 International License.

Read Full License

Version of Record: A version of this preprint was published at BMC Cancer on May 24th, 2021. See the published version at https://doi.org/10.1186/s12885-021-08244-2. 


\section{Abstract}

Background: Renal cancer is a common malignant tumor with an increasing incidence rate.

Methods: In this study, based on the gene expression profiles, we analyzed the compositions of tumorinfiltrating immune cells (TIICs) in renal cancer and paracancerous samples using CIBERSORT. The proportions of 22 TIICs subsets in 122 paired renal carcinoma and paracancerous samples, and 224 Wilms tumor (WT) samples varied between intragroup and intergroup.

Results: After analyzed the difference of TIICs composition between renal cancer and paired paracancerous samples, we found that M0 macrophages and CD8 T cells were significantly elevated, while naive B cells were significantly decreased in renal cancer samples compared with paracancerous samples. Survival analysis showed that high overall TIICs proportion, the low proportion of resting mast cells and the high proportion of activated memory CD4 T cells were associated with poor prognosis of renal cancer patients. In addition, 3 clusters were identified by hierarchical clustering analysis, and they presented a distinct prognosis. Cluster 1 had superior survival outcomes, while cluster 2 had an inferior survival outcome.

Conclusions: Our study indicated that overall TIICs proportion, certain TIICs subset proportion, including resting mast cells and activated memory CD4 T cells, and distinct cluster patterns were associated with the prognosis of renal cancer, which was significant for the clinical surveillance and treatment of renal cancer.

\section{Background}

Renal cancer is a common malignant tumor with an increasing incidence rate over time (Corgna et al. 2007; Weir et al. 2016). Wilms tumor (WT), also known as nephroblastoma, is the second most common solid abdominal organ tumor in children and the most frequent primary malignant renal cancer (Ehrlich 2017). It accounts for $6 \%$ of pediatric cancers, and an estimated $95 \%$ of renal cancer is WT among children (Barber et al. 2007). Clinically, WT or nephroblastoma is a common childhood tumor that is intimately linked to early kidney development and is often associated with persistent embryonic renal tissue and other kidney abnormalities, which is different from other types of renal cancer (Rivera and Haber. 2005). As an embryonic tumor of the kidney, WT arises from metanephric mesenchyme and presents high a incidence rate among 2-3-year-old children (Perotti et al. 2007). The treatment of WT is determined by several factors, such as genetic, pathologic, and demographic factors, and current therapies for WT consist of surgery, chemotherapy, and radiation therapy (Ehrlich 2017; Barber et al. 2011). Although the survival of WT has improved with the development of treatment strategies, the prognosis-related factors, including hematogenous and pulmonary metastasis, relatively high recurrence rate, and late effects, make the prospects of treatment noteworthy (Njuguna et al. 2017; Davidoff 2012). Therefore, further investigation of the biological processes and underlying molecular mechanisms of WT may facilitate the prognosis improvement of patients. 
Currently, increasing evidence has proved that the occurrence and development of tumors are associated with tumor cells as well as tumor microenvironment (Liu et al. 2017). The tumor microenvironment is composed of complex components, such as mesenchymal stem cells, fibroblasts, and immune cells (Isaac and Sharma 2015). Among them, tumor-infiltrating immune cells (TIICs) are the pivotal components and are considered the leading players of the tumor microenvironment (Ino et al. 2013). Accumulating studies have shown that TIICs are closely associated with the development and survival outcome of cancers. For example, infiltrated macrophages were found to promote the progression of prostate cancer, and NK cells showed anti-tumor effects against tumor development (Gannon et al. 2009). In colorectal cancer, TIICs were associated with patients' clinical outcomes and considered key signatures for prognosis (Caro et al. 2014). The immunohistologic features and immunotype of TIICs played a crucial role in metastatic melanoma and were related to the survival outcome (Erdag et al. 2012). In addition, dysfunctional infiltrating lymphocytes were observed in renal cell carcinoma (RCC), immunogenic renal cancer (Thompson et al. 2007). It was indicated that tumors might exert an impairment effect on the immune system, and more studies on the associations between TIICs and tumor occurrence and prognosis should be helpful for the application of immunotherapeutic strategies on renal cancer.

Immunohistochemistry is a commonly used method to evaluate the TIICs in tumors. However, the results are often inaccurate due to the extensive-expression of markers in non-immune cells (Liu et al. 2017). In our research, the CIBERSORT algorithm was adopted to assess the TIICs subsets in renal cancer based on gene expression profiles retrieved from The Cancer Genome Atlas (TCGA). Meanwhile, we also analyzed the relationship of TIICs fraction and immune patterns with the survival outcome of renal cancer to explore the prognostic values of TIICs fraction and immune clusters in renal cancer.

\section{Methods}

\subsection{Data source}

We downloaded the gene expression profiles of renal cancer from the Gene Expression Omnibus (GEO, https://www.ncbi.nlm.nih.gov/geo/, Affymetrix HG-U133A platform) and The Cancer Genome Atlas (TCGA, www.cancergenome.nih.gov, Illumina HiSeq platform). The TCGA dataset was composed of 889 renal cancer samples and 128 paracancerous samples, including chromophobe renal cell carcinoma, kidney renal clear cell carcinoma, and kidney renal papillary cell carcinoma samples. The GEO dataset (access no. GSE31403) included 224 Wilms tumor (WT) samples.

\subsection{Tumor-infiltrating immune cells calculation}

CIBERSORT (http://cibersort.stanford.edu), a deconvolution algorithm based on gene expression profiles (Xiong et al. 2018), was used to calculate the relative proportions of 22 tumor-infiltrating immune cells (TIICs) subsets. CIBERSORT is able to evaluate the composition of TIICs with 547 barcode gene expression values, using $P$-value as a parameter for measurement of the confidence in results. 
In addition, In the complex cancer immune microenvironment, cytotoxic T cells (Tc) and NK cells are two main effector cell types that can attack tumor cells directly. Upon exposure to transformed cells, cytotoxic T cells and NK cells secrete granzymes (a family of serine proteases) and perforin (a pore-forming protein) that will ultimately lead to target cell death. Thus, the local immune cytolytic activity can be quantified based on the transcript levels of perforin (PRF1) and granzyme A (GZMA). The first cytotoxin polymerizes and creates a channel in the membrane of the target cell. Through these pores, granzymes will then enter the cytoplasm and trigger a caspase cascade, composed of cysteine proteases that will ultimately lead to apoptosis (Trapani and Smyth. 2002). GZMA is a tryptase that induces caspaseindependent programmed cell death, and PRF1 serves as a pore-forming enzyme that regulates entry of granzymes into target cells (Chowdhury and Lieberman. 2008). Accordingly, it has been well-identified that the mean expression levels of genes GZMA and PRF1 represent the immune cytolytic activity of immune cells, which also reflect the fraction of TIICs (Constantinos ea al. 2018; Narayanan et al. 2018; Gu et al. 2019; Tian et al. 2018; Gong et al. 2019; Rooney et al. 2015). Therefore, the expression levels of GZMA and PRF1 were determined to comprehensively assess the immune cytolytic activity and evaluate TIICs composition.

\subsection{Survival analysis}

All 1241 samples were grouped according to results of CIBERSORT with $P$-values greater than or equal to 0.05 and less than 0.05 , then the proportion of samples in each group and the average expression levels of genes GZMA and PRF1 were calculated. Seven samples of patiens without survival information were excluded, then based on kaplan-Meier method, survival analyses of the 1,010 samples from TCGA, which were also stratified by $P$-value of 0.05 were performed by survival package (https://cran.rproject.org/web/packages/survival/) and survminer package (https://cran.r-

project.org/web/packages/survminer/). With the relative proportions of $22 \mathrm{TIICs}$ subsets as continuous variables, we calculated the hazard ratios (HR) using Cox regression analysis. We performed survival analyses to explore the effects of significant factors on survival outcomes.

\subsection{Clustering analysis}

Based on K-mean method, cluster the samples with the relative content of immune infiltrated cells by $\mathrm{R}$ software v3.5.2.

\subsection{Statistical analyses}

We used R language to calculate the proportions of 22 TIICs subpopulations in the 1,241 samples from TCGA and GEO datasets. The correlation of the results from different datasets was analyzed by Pearson correlation analysis. The compositions of TIICs subpopulations in 122 paired cancer and paracancerous samples from TCGA were computed, and the difference in compositions was further analyzed. Besides, the fraction of TIICs subsets in WT samples from GEO was also calculated. 
Then we stratified the 1,241 samples by $P$-value of 0.05 , and calculated the compositions of TIICs subsets as well as mean expression values of GZMA and PRF1 in the groups with $P \geq 0.05$ and $P<0.05$ respectively.

\section{Results}

\subsection{Performance of CIBERSORT for TIICs evaluation in renal cancer}

The TIICs composition was analyzed by CIBERSORT. As shown in Figure 1A, the samples from GEO and TCGA presented distinct proportions of 22 TIICs subsets. As the renal cancer samples in GEO were all WT patients, we speculated that the TIICs in WT samples were significantly different from those in other renal cancer samples. Then we analyzed the correlation of immune cell proportions in renal cancer samples from TCGA and GEO. It was revealed that the renal cancer samples from the two datasets had highly consistent immune cell proportions (Figure 1B), illustrating that CIBERSORT could evaluate the fraction of TIICs independently of data sources and platforms.

\subsection{Landscape of TIICs in renal cancer}

The compositions of TIICs subsets in 122 paired paracancerous and renal cancer samples from the TCGA and 224 WT samples from GEO were calculated by CIBERSORT, respectively. As shown in Figure 2A-C and Table S1 the intragroup and intergroup differences in TIICs fractions were manifest. Thus, we inferred that the proportion of TIICs subsets was an inherent characteristic which varied significantly among different individuals. In addition, we analyzed the difference in TIICs composition between renal cancer and paired paracancerous samples. As shown in Figure 2D, M0 macrophages and CD8 T cells were significantly elevated, while naive B cells were significantly decreased in renal cancer samples compared with those in paired paracancerous samples, and the results were basically consistent with previous researches (Zhong et al. 2019; Rohr-Udilova et al. 2018).

\subsection{P-value of CIBERSORT represents the overall proportion of TIICS}

It should be noted that instead of determining the actual values, CIBERSORT only calculates the relative ratios of TIICs subsets, which contributes to the dependency of results on each other. Therefore, we further analyzed the association between the $P$-value provided by CIBERSORT and TIICs composition. CIBERSORT $P$-value $<0.05$ correlate with higher immune cell infiltrates, while no significantly different of the $P$-value $\geq 0.05$ (Raza ea al. 2016; Yongfu et al. 2018). Figure 3A showed that the proportions of samples with $P$-value $<0.05$ and $P$-value $\geq 0.05$ in TCGA and GEO were obviously different.

It has been well proved that the mean expression values of GZMA and PRF1 represent the immune cytolytic activity and are positively associated with TIICs proportions (Constantinos et al. 2018; Narayanan et al. 2018; Gu et al. 2019; Tian et al. 2018; Gong et al. 2019; Rooney et al. 2015). Accordingly, we futher evaluated the mean expression values, which represented the immune cytolytic activity in the system. After analyzing their mean expression values, we found that the samples with $P$-value $<0.05$ had 
higher immune cytolytic activity in both TCGA and GEO cohorts $(p<2.22 \mathrm{e}-16$ and $p=0.043$, respectively, Figure 3B and $3 \mathrm{C}$ ). These results indicated that the proportion of TIICs in samples with $P$-value $<0.05$ was higher in comparison to samples with $P$-value $\geq 0.05$.

3.4 Overall TIICs proportion, resting mast cells and activated memory CD4 T cells are associated with prognosis of renal cancer

To investigate the effects of overall TIICs proportion or 22 individual TIICs subset on renal cancer prognosis, the survival analyses of 1,010 renal cancer samples with survival information were performed. The survival curve of renal cancer samples stratified by $P$-value of 0.05 showed that samples with $P$ value $<0.05$ presented inferior survival outcome in comparison to samples with $P$-value $\geq 0.05$ ( $p<$ 0.0001 , Figure 3D). It was suggested that high TIICs proportions might be associated with poor prognosis of renal cancer patients.

Subsequently, to further explore the effects of 22 individual TIICs subset on renal cancer prognosis, the univariate Cox regression analysis was conducted with 22 TIICs subsets as continuous variables. The relevant 95\% confidence intervals and Hazard Ratios (HRs) were shown in Figure 4A. It was found that activated memory CD4 T cells and resting mast cells were significantly associated with the prognosis of renal cancer patients $(H R=9.4 e+05, p=0.004$ and $H R=3.7 e-03, p=0.034$, respectively). The survival curves showed a low proportion of resting mast cells and a high proportion of activated memory CD 4 T cells were related to poor prognosis of renal cancer patients (both $p<0.0001$ ).

\subsection{Different immune clusters are associated with the prognosis of renal cancer}

The above results indicated TIICs alteration might affect the prognosis of renal cancer patients. Therefore, we speculated whether different immune clusters could be identified with the TIICs data. Firstly, the optimal number of clusters was determined as 3 using the within cluster sum of square errors (WSS) method (Figure S1). Subsequently, the hierarchical clustering of the samples was conducted by the Euclidean distance model (Figure 5A). After analyzed the association of different immune patterns with prognosis, cluster 1 exhibited superior survival outcomes, while cluster 2 exhibited inferior survival outcomes ( $p=0.0057$, Figure $5 B$ ). Moreover, the compositions of 22 TIICs subsets is significantly different among the cluster 1 , cluster 2 , and cluster 3 (Figure 5 C). In additional, the immunes cell type abundances differ between clusters was revealed in violin plot Figure S2 and clinical differences of the three clusters was showed in the Figure S3. The results suggested that the relative proportion of 20 types of immune cells was significantly different in 3 clusters $(P<0.05$, ANOVA). In Cluster1, with the best prognosis, the relative proportion of these 4 types of immune cells, including T cells CD4 memory resting, Macrophages M1, Mast cells resting and Monocytes, and Monocyte, were significantly higher than that of the other two clusters.

\section{Discussion}


The tumor microenvironment is considered as a complex "society", and exerts a regulatory effect on tumor progression with the participation of multiple cell types and extracellular matrix (Albini et al. 2010). The various cell types in tumor microenvironment include mesenchymal stem cells, fibroblasts, endothelial cells, and immune cells (Ungefroren et al. 2011). It is believed that the TIICs are indispensable members of the tumor microenvironment and reflect the host immune reaction to tumors (Ino et al. 2013). Accumulating evidence have proved that TIICs are associated with tumor invasion, metastasis, prognosis, and response to therapy (Jiang et al. 2011; Jochems and Schlom. 2011). Given their important role, comprehensive researches on the TIICs in the tumor microenvironment may provide novel therapeutic approaches for tumors.

Numerous efforts have been devoted to exploring the specific role of TIICs in renal cancer by multiple approaches. Jensen HK et al. evaluated the TIICs in localized renal cell carcinoma by immunohistochemistry and found that neutrophils were independent prognostic signatures of survival outcome for localized renal cell carcinoma (Jensen et al. 2009). Donskov F et al. identified the positive correlation of CD57+ NK cells and negative correlation of neutrophils with the prognosis of metastatic renal cell carcinoma by immunohistochemistry (Donskov 2006). Webster WS et al. proved that the infiltrated mononuclear cells were able to predict the survival outcome of renal cell carcinoma patients independently through hematoxylin and eosin staining and flow cytometric analysis (Webster et al. 2006). Unlike the above researches, the TIICs in renal cancer were analyzed using a distinct approach in our study. We assessed the 22 TIICs subpopulations composition of renal cancer and paracancerous samples from TCGA and GEO using CIBERSORT algorithm based on their gene expression profiles. The TIICs subsets proportions of renal cancer samples from TCGA and GEO showed consistent results after correlation analysis. It was suggested that CIBERSORT was able to assess the composition of the TIICs subset, which was independent of data platforms and sources. Besides, CIBERSORT could be performed to characterize cell heterogeneity using RNA mixtures from nearly any source (Newman et al. 2015). Many researchers used CIBERSORT for estimating the infiltration of immune cells, and there is no differences between using CIBERSORT on microarray datasets (Zhou et al. 2018; Stahl et al. 2019; Xu et al. 2019) and RNA sequencing datasets (Yazdani et al. 2018; Chaumette et al. 2020; Diaz-Mejia et al. 2019), even on both microarray datasets and RNA sequencing datasets (Wang et al. 2020). Meanwhile, it has been reported that CIBERSORT has been used in several previous studies to analyzed the TIICs in the renal cell carcinoma, which have identified that $C D 8+T$ cells were associated with prolonged overall survival and the potential biomarker relaed to CD8+ T cells, respectively (Zhang et al. 2019; Lin et al. 2020). However, several researches reported that elevated CD8+ T cells were negatively related to prognosis in the patients with glioma (Zhong et al. 2019) and hepatocellular carcinoma (Rohr-Udilova et al. 2018). Besides, a different method of ESTIMATE algorithm has also been applied to analyze prognostic microenvironment-related genes and stromal and immune scores in Clear Cell Renal Cell Carcinoma (Chen et al. 2019). In this study, our CIBERSORT analysis identified the landscape of TIICs in renal cancer and demonstated high TIICs proportions might be associated with poor prognosis of renal cancer patients. These data providing new evidence of the application of CIBERSORT in renal cancer and provide valuable information of TIICs in renal cancer patients. 
Then we investigated the association of 22 individual TIICs subpopulation with the survival outcome of renal cancer patients by Cox regression analysis and survival analysis. The results showed that elevated proportion of activated memory CD4 T cells and decreased proportion of resting mast cells were associated with poor prognosis of renal cancer. It was known that the activated memory CD4 T cells could secret interleukin (IL) 17, a proinflammatory cytokine that promotes the proliferation and growth of cervical cancer (Tartour et al. 1999). It has been reported that IL-17 is able to promote the development of colorectal cancer and associate with poor prognosis (Liu et al. 2011). In the present study, a high proportion of activated memory CD $4 \mathrm{~T}$ cells were related to poor prognosis of renal cancer patients. Hence, we speculated that the increased proportion of activated memory CD4 T cells contributed to inferior survival outcomes in renal cancer through enhanced secretion of IL-17, which might be one of the possible mechanisms and needed to be validated further in the future investigations. Mast cells, which reside in vascularized tissues, have two states, including resting condition and activated condition (Carla et al. 2015). They are involved in multiple tumor-related processes. In thyroid cancer, thyroid cancer cells activated the mast cells, which could secrete extensive proinflammatory, angiogenic, and growth factors and exert protumorigenic effects (Carla et al. 2015). We inferred that the decreased proportion of resting mast cells, which reflected the enhancement of activated mast cells activities, was associated with a poor prognosis due to the protumorigenic effects of activated mast cells. In addition, a previous study showed a four immune-related genes signature based on CXCL2, SEMA3G, PDGFD, and UCN is closely associated with the prognosis of renal clear cell carcinoma (Gao et al. 2020). In this study, we identified that TIICs proportion is closely correlated with the prognosis of renal cancer, which was diffent from the previous study.

In addition, a total of 3 immune clusters was identified by hierarchical clustering analysis. After analyzing their fractions, we found that the proportions of TIICs subpopulations presented remarkable difference in the different clusters. The survival analysis revealed that the survival outcomes were significantly diffenrt among these 3 clusters, in which cluster 1 exhibited superior survival outcomes, while cluster 2 exhibited inferior survival outcomes. These findings indicated that immune infiltrate is heterogeneously different in the renal cancer patients and the difference of immune infiltrate is closely associated with the prognosis of of renal cancer patients.

\section{Conclusions}

In summary, the analysis of 22 TIICs proportions in renal cancer samples showed elevated activated memory CD 4 T cells proportion, and decreased resting mast cells proportion predicted poor prognosis in renal cancer. Different immune clusters also presented distinct survival outcomes. The results might unveil novel prognosis prediction and immunotherapeutic strategies on renal cancer.

\section{Declarations}

Ethics approval and consent to participate: Not Applicable. 
Consent for publication: Not Applicable.

Availability of data and material: All data generated or analyzed during this study are included in this published article.

Competing interest: The authors declare that there are no conflicts of interest.

Funding: Not Applicable.

Authors' contributions: All authors read and approved the manuscript. LC and LY made substantial contributions to conception and design, acquisition of data, analysis and interpretation of data; ZQ and $\mathrm{JL}$ performed the experiments; XW have been involved in drafting the manuscript or revising it critically for important intellectual content; KM given final approval of the version to be published. XL agreed to be accountable for all aspects of the work in ensuring that questions related to the accuracy or integrity of any part of the work are appropriately investigated and resolved.

Acknowledgments: Not Applicable.

\section{References}

Albini A, Magnani E, Noonan DM (2010) The tumor microenvironment: biology of a complex cellular and tissue society. The quarterly journal of nuclear medicine and molecular imaging: official publication of the Italian Association of Nuclear Medicine (AIMN) [and] the International Association of Radiopharmacology (IAR), [and] Section of the Society of 54:244-248

Barber TD, Derinkuyu BE, Wickiser J, Joglar J, Koral K, Baker LA (2011) Wilms tumor: preoperative risk factors identified for intraoperative tumor spill. The Journal of Urology 185:1414-1418

Carla V, Nella P, Federica L, Gianni M (2015) Tumor-associated mast cells in thyroid cancer. International Journal of Endocrinology 2015:1-8

Caro GD Bergomas F, Grizzi F, Doni A, Bianchi P, Malesci A (2014) Occurrence of tertiary lymphoid tissue is associated with t-cell infiltration and predicts better prognosis in early-stage colorectal cancers. Clinical Cancer Research 20:2147-2158

\section{Chaumette B, Kebir O, Dion PA, Rouleau GA, Krebs MO (2020) Reliability and correlation of mixture cell correction in methylomic and transcriptomic blood data 13:74.}

\section{Chen B, Chen W, Jin J, Wang X, He Y (2019) Data mining of prognostic microenvironment-related genes in clear cell renal cell carcinoma: a}




\section{study with tcga database. Disease markers 1-11.}

\section{Chowdhury D, Lieberman J (2008) Death by a thousand cuts: granzyme pathways of programmed cell death. Annu Rev Immunol 26:389-420.}

Constantinos R, Dimitrios C, Anestis M, Efstathiades C, Dimopoulos C, Zaravinos A (2018) The Expression and Prognostic Impact of Immune Cytolytic Activity-Related Markers in Human Malignancies: A Comprehensive Meta-analysis. Frontiers in Oncology 8:27.

Corgna E, Betti M, Gatta G, Roila F, Mulder PHMD (2007) Renal cancer. Critical Reviews in Oncology/Hematology 64:247-262

Davidoff AM (2012) Wilms' tumor. Advances in Pediatrics 59:247-267

Diaz-Mejia JJ, Meng EC, Pico AR, MacParland SA, Ketela T, Pugh TJ (2019) Evaluation of methods to assign cell type labels to cell clusters from single-cell RNA-sequencing data 8:ISCB Comm J-296.

Donskov F (2006) Impact of immune parameters on long-term survival in metastatic renal cell carcinoma. Journal of Clinical Oncology 24:1997-2005

Erdag G, Schaefer JT, Smolkin ME, Deacon DH, Shea SM, Dengel LT (2012) Immunotype and immunohistologic characteristics of tumor-infiltrating immune cells are associated with clinical outcome in metastatic melanoma. Cancer Research 72:1070-1080

Ehrlich PF (2017) Wilms Tumor. Fundamentals of Pediatric Surgery. Springer International Publishing.

Gao X, Yang J, Chen Y (2020) Identification of a four immune-related genes signature based on an immunogenomic landscape analysis of clear cell renal cell carcinoma. Journal of Cellular Physiology.

Gannon PO, Poisson AO, Delvoye N, Réjean Lapointe, Mes-Masson AM, Saad F (2009) Characterization of the intra-prostatic immune cell infiltration in androgen-deprived prostate cancer patients. Journal of Immunological Methods 348: 9-17

Gong Z, Jia Q, Chen J, Diao X, Gao J, Wang X (2019) Impaired cytolytic activity and loss of clonal neoantigens in elderly patients with lung adenocarcinoma. Journal of Thoracic Oncology 14:857-866.

Gu XL, Boldrup LD, Coates PJ, Fahraeus R, Wang LX, Wilms TB (2019) High immune cytolytic activity in tumor-free tongue tissue confers better prognosis in patients with squamous cell carcinoma of the oral tongue. The Journal of Pathology: Clinical Research 5:240-247.

Ino Y, Yamazaki-Itoh R, Shimada K, Iwasaki M, Kosuge T, Kanai Y (2013) Immune cell infiltration as an indicator of the immune microenvironment of pancreatic cancer. British Journal of Cancer 108:914-923 
Isaac A, Sharma B (2015) Modulation of the tumor microenvironment for cancer treatment: a biomaterials approach. Journal of Functional Biomaterials 6:81-103

Jiang B, Mason J, Jewett A, Liu ML, Man YG (2013) Tumor-infiltrating immune cells: triggers for tumor capsule disruption and tumor progression?. International journal of medical sciences 10:475-497

Jensen HK, Donskov F, Marcussen N, Nordsmark M, Lundbeck F, Von DMH (2009) Presence of intratumoral neutrophils is an independent prognostic factor in localized renal cell carcinoma. Journal of Clinical Oncology 27:4709-4717

Jochems C, Schlom J (2011) Tumor-infiltrating immune cells and prognosis: the potential link between conventional cancer therapy and immunity. Experimental Biology and Medicine 236:567-579

Lin JX, Yu M, Xu X, Wang YT, Xing HT, An J (2020) Identification of biomarkers related to CD8 + T cell infiltration with gene co-expression network in clear cell renal cell carcinoma. Aging (Albany NY) 12:36943712.

Liu J, Duan Y, Cheng X, Chen X, Xie W, Long H (2011) II-17 is associated with poor prognosis and promotes angiogenesis via stimulating vegf production of cancer cells in colorectal carcinoma. Biochemical \& Biophysical Research Communications 407:348-354

Liu X, Wu S, Yang Y, Zhao M, Zhu G, Hou Z (2017) The prognostic landscape of tumor-infiltrating immune cell and immunomodulators in lung cancer. Biomedicine \& Pharmacotherapy 95:55-61

Narayanan S, Kawaguchi T, Yan L, Peng X, Qi Q, Takabe K (2018) Cytolytic activity score to assess anticancer immunity in colorectal cancer. Annals of Surgical Oncology 25:2323-2331.

Newman AM, Liu CL, Green MR, Gentles AJ, Feng W, Xu Y (2015) Robust enumeration of cell subsets from tissue expression profiles. Nature Methods 12:453-457.

Njuguna F, Martijn HA, Kuremu RT, Saula P, Kirtika P, Olbara G (2017) Wilms tumor treatment outcomes: perspectives from a low-income setting. Journal of Global Oncology 3:555-562

Perotti D, Hohenstein P, Bongarzone I, Maschietto M, Weeks M, Radice P (2013) Is wilms tumor a candidate neoplasia for treatment with wnt/ $\beta$-catenin pathway modulators? -a report from the renal tumors biology-driven drug development workshop. Molecular Cancer Therapeutics 12:2619-2627

Raza AH, Leon C, Pharoah PDP, Florian M, Carlos C, Marc L (2016) Patterns of immune infiltration in breast cancer and their clinical implications: a gene-expression-based retrospective study. Plos Medicine 13:e1002194.

Rivera MN, Haber DA (2005) Wilms' tumour: connecting tumorigenesis and organ development in the kidney. Nature Reviews Cancer 5:699. 
Rohr-Udilova N, Klinglmüller F, Schulte-Hermann R, Stift J, Herac M, Salzmann M, et al. (2018) Deviations of the immune cell landscape between healthy liver and hepatocellular carcinoma. Scientific Reports 8: 6220.

Rooney M, Shukla S, Wu C, Getz G, Hacohen N (2015) Molecular and genetic properties of tumors associated with local immune cytolytic activity. Cell 160:48-61

Stahl D, Gentles AJ, Thiele R, Ines Gütgemann (2019) Prognostic profiling of the immune cell microenvironment in ewing's sarcoma family of tumors. Oncolmmunology 8:e1674113.

Tartour E, Fossiez F, Joyeux I, Galinha A, Gey A, Claret E (1999) Interleukin 17, a t-cell-derived cytokine, promotes tumorigenicity of human cervical tumors in nude mice. Cancer Research 59:3698

Thompson RH, Dong H, Lohse CM, Leibovich BC, Blute ML, Cheville JC (2007) Pd-1 is expressed by tumor-infiltrating immune cells and is associated with poor outcome for patients with renal cell carcinoma. Clinical Cancer Research 13: 1757-1761

Tian T, Ji W, Yan H, Haoran L, Feng G, Youdong P (2018) A comprehensive survey of immune cytolytic activity-associated gene co-expression networks across 17 tumor and normal tissue types. Cancers 10:307.

Trapani JA, Smyth MJ (2002) Functional significance of the perforin/granzyme cell death pathway. Nat Rev Immunol 2:735-747.

Ungefroren H, Sebens S, Seidl D, Lehnert H, Hass R (2011) Interaction of tumor cells with the microenvironment. Cell Communication and Signaling 9:18

Wang X, Wu Y, Wen D, Wu LY, Yang H (2020) An individualized immune prognostic index is a superior predictor of survival of hepatocellular carcinoma. Medical ence monitor: international medical journal of experimental and clinical research 26:e921786.

Webster WS, Lohse CM, Thompson RH, Dong H, Frigola X, Dicks DL (2006) Mononuclear cell infiltration in clear-cell renal cell carcinoma independently predicts patient survival. Cancer 107

Weir HK, Johnson CJ, Ward KC, Coleman MP (2016) The effect of multiple primary rules on cancer incidence rates and trends. Cancer Causes \& Control 27:377-390.

Xiong Y, Wang K, Zhou H, Peng L, You W, Fu Z (2018) Profiles of immune infiltration in colorectal cancer and their clinical significant: A gene expression-based study. Cancer Med 7:4496-4508

Xu F, Zhang H, Chen J, Lin L, Chen Y (2019) Immune signature of $t$ follicular helper cells predicts clinical prognostic and therapeutic impact in lung squamous cell carcinoma. International Immunopharmacology 81:105932. 
Yazdani S, Callemeyn J, Gazut S, Lerut E, De Loor H, Wevers M (2018) Natural killer cell infiltration is discriminative for antibody-mediated rejection and predicts outcome after kidney transplantation. Kidney International 95:188-198.

Yongfu X, Kang W, He Z, Linglong P, Wenxian Y, Zhongxue F (2018) Profiles of immune infiltration in colorectal cancer and their clinical significant: a gene expression-based study. Cancer Medicine 7:44964508.

Zhang S, Zhang E, Long J, Hu Z, Peng J, Liu L (2019) Immune infiltration in renal cell carcinoma. Cancer Science 110:1564-1572.

Zhong QY, Fan EX, Feng GY, Chen QY, Zhang GH (2019) A gene expression-based study on immune cell subtypes and glioma prognosis. BMC Cancer 19:1116.

Zhou R, Zhang JW, Zeng DQ, Sun HY, Rong XX, Shi M (2018) Immune cell infiltration as a biomarker for the diagnosis and prognosis of stage i-iii colon cancer. Cancer Immunology Immunotherapy 68:433-442.

\section{Figures}
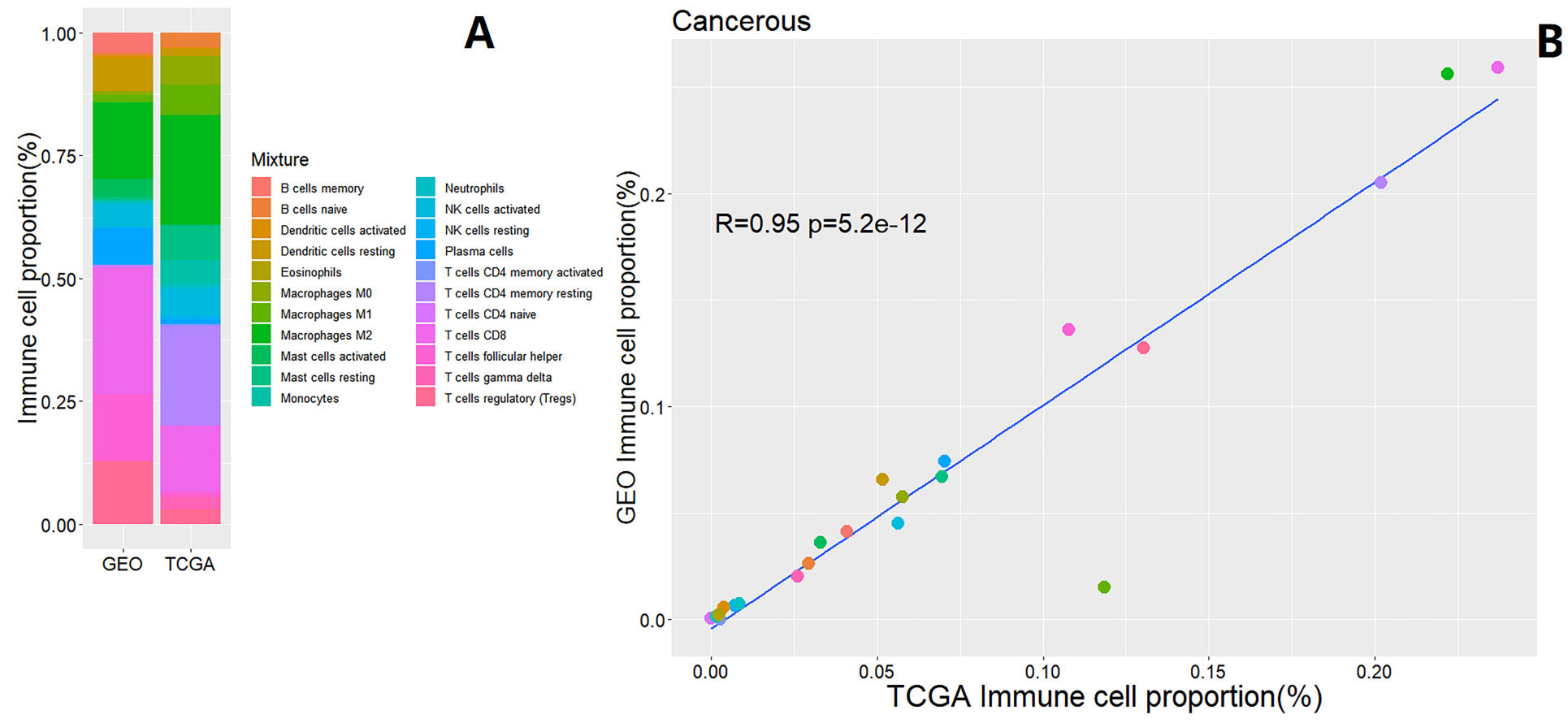

\section{Figure 1}

Renal cancer samples from GEO and TCGA had highly consistent immune cell proportion. (A) TIICs compositions of renal cancer and paracancerous samples from TCGA and GEO analyzed by CIBERSORT.

(B) Proportions of TIICs subsets in renal cancer samples from TCGA and GEO. 

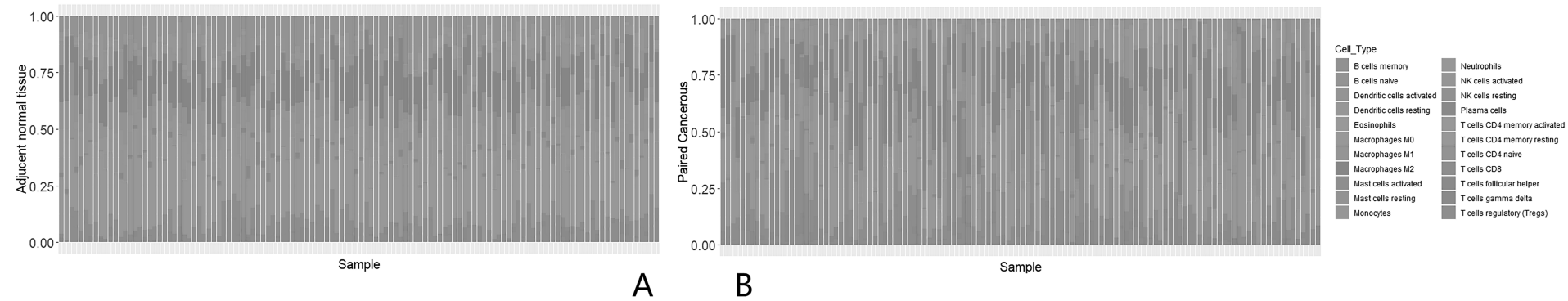

A

B

C D
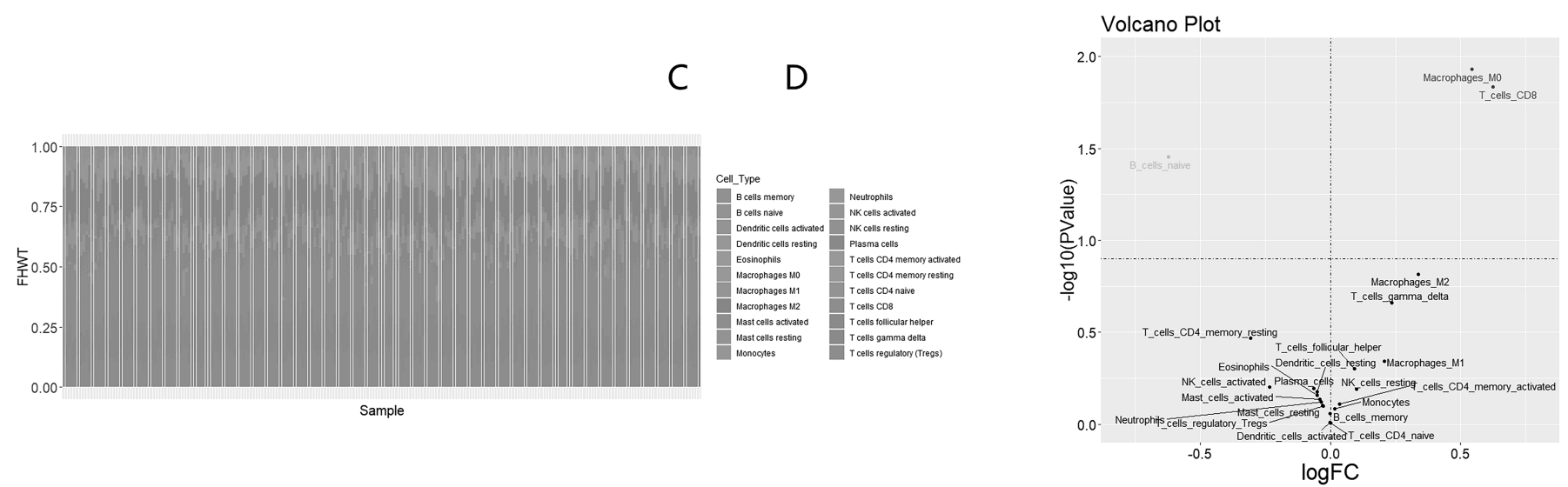

\section{Figure 2}

Obvious intragroup and intergroup differences in TIICs fraction were observed among paired renal cancer and paracancerous samples from TCGA, and WT samples from GEO. (A) Immune infiltration in paired paracancerous samples. (B) Immune infiltration in paired renal cancer samples. (C) Immune infiltration in WT samples. (D) Volcano plot of TIICs subsets proportions between paired renal cancer and paracancerous samples. Compared with paired paracancerous samples, M0 macrophages and CD8 T cells were significantly elevated, while naive B cells were significantly decreased in renal cancer samples.
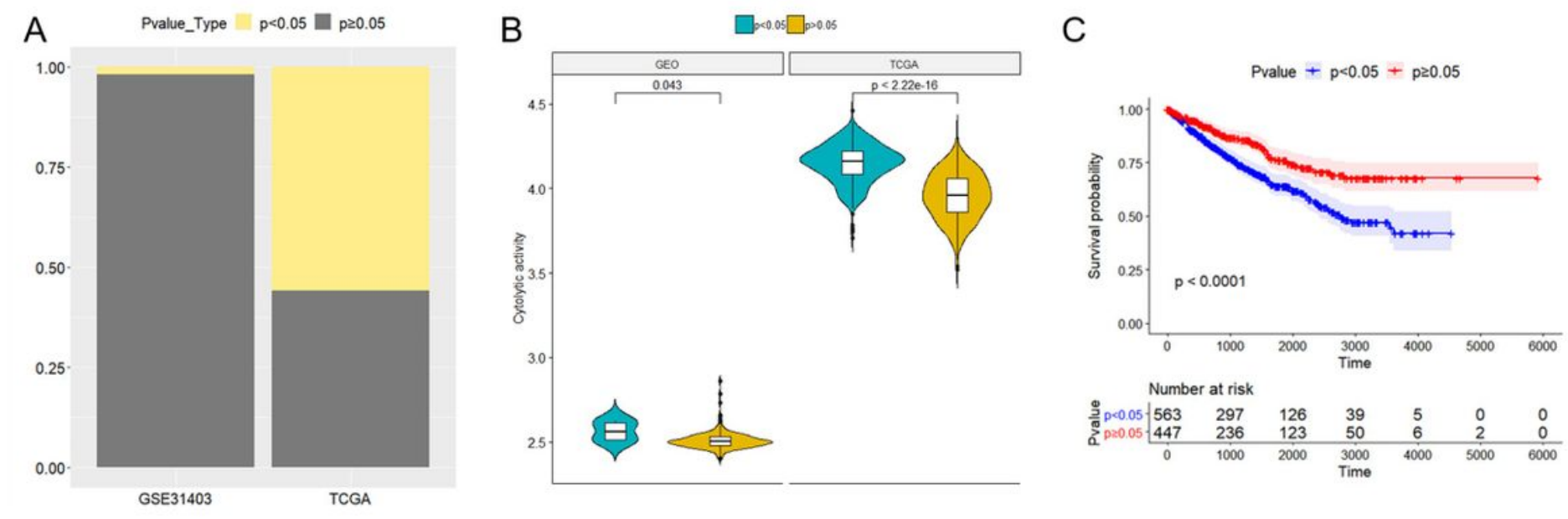

\section{Figure 3}

P-value of CIBERSORT represented the overall proportion of TIICs. (A) Proportions of samples with Pvalue $<0.05$ and P-value $\geq 0.05$ in GEO and TCGA datasets. (B) Immune cytolytic activity of samples with P-value $<0.05$ and P-value $\geq 0.05$ in TCGA and GEO cohort. (C) Survival curves of samples stratified 
by P-value of 0.05 . Compared with renal cancer patients with P-value $\geq 0.05$, patients with P-value $<0.05$ had poor survival outcome $(p<0.0001)$.

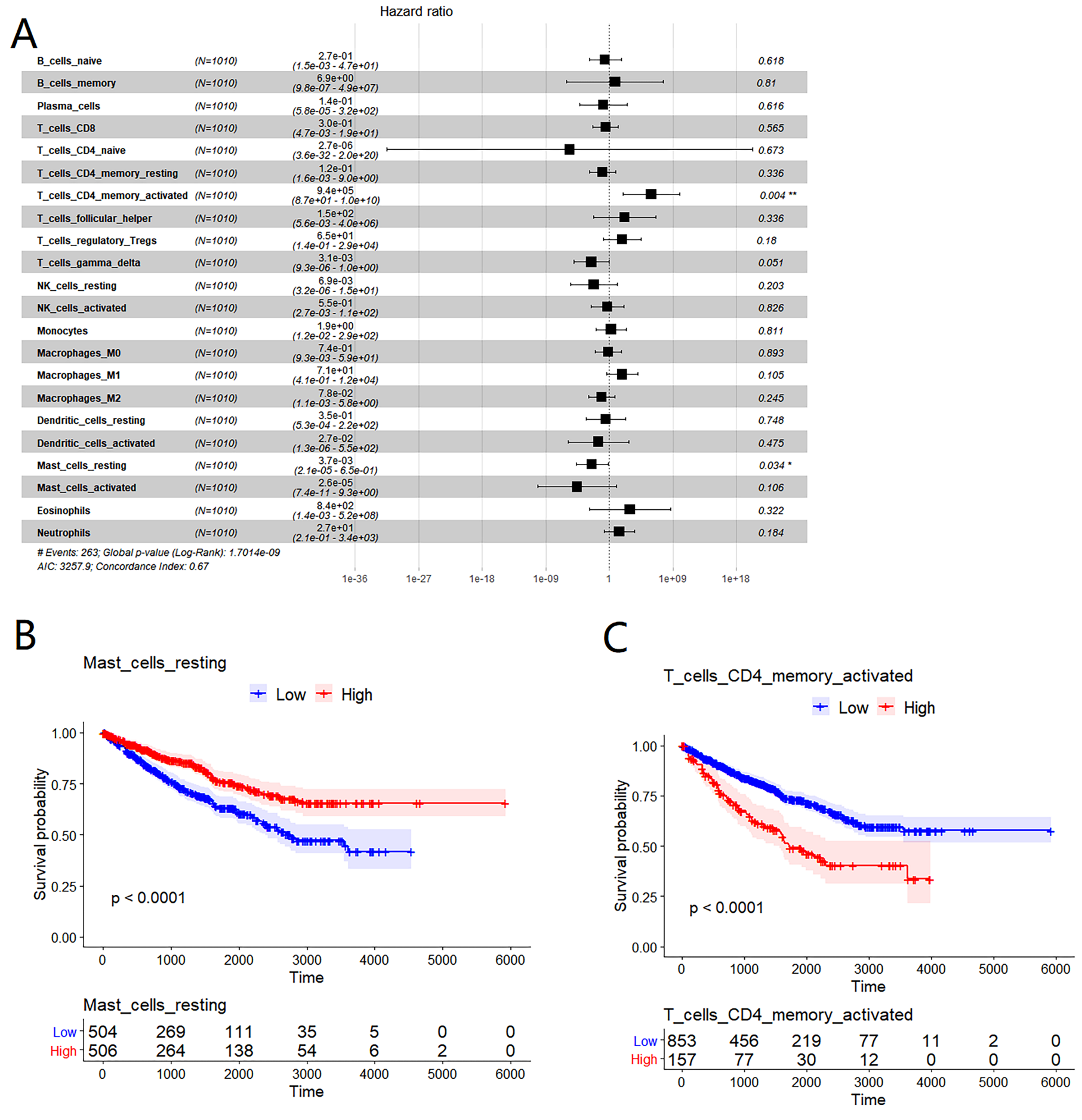

\section{Figure 4}

Decreased resting mast cells and elevated activated memory CD4 T cells were associated with poor prognosis of renal cancer patients. (A) Association of 22 TIICs subsets with survival outcomes of renal cancer patients. * and $* \star$ indicated statistical significance. (B) Survival curve of resting mast cells 
stratified by median proportion. (C) Survival curve of activated memory CD4 T cells stratified by median proportion.
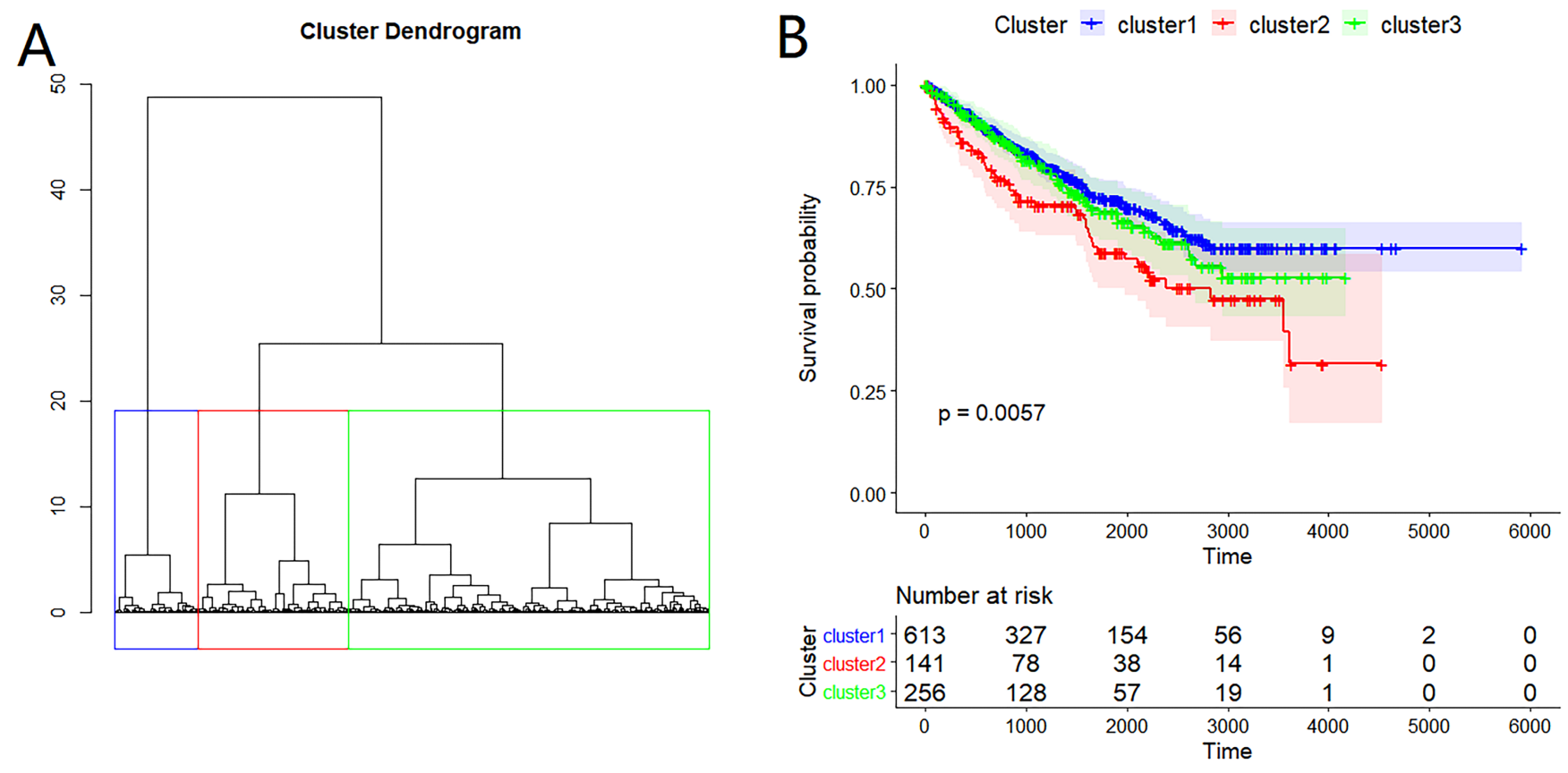

C
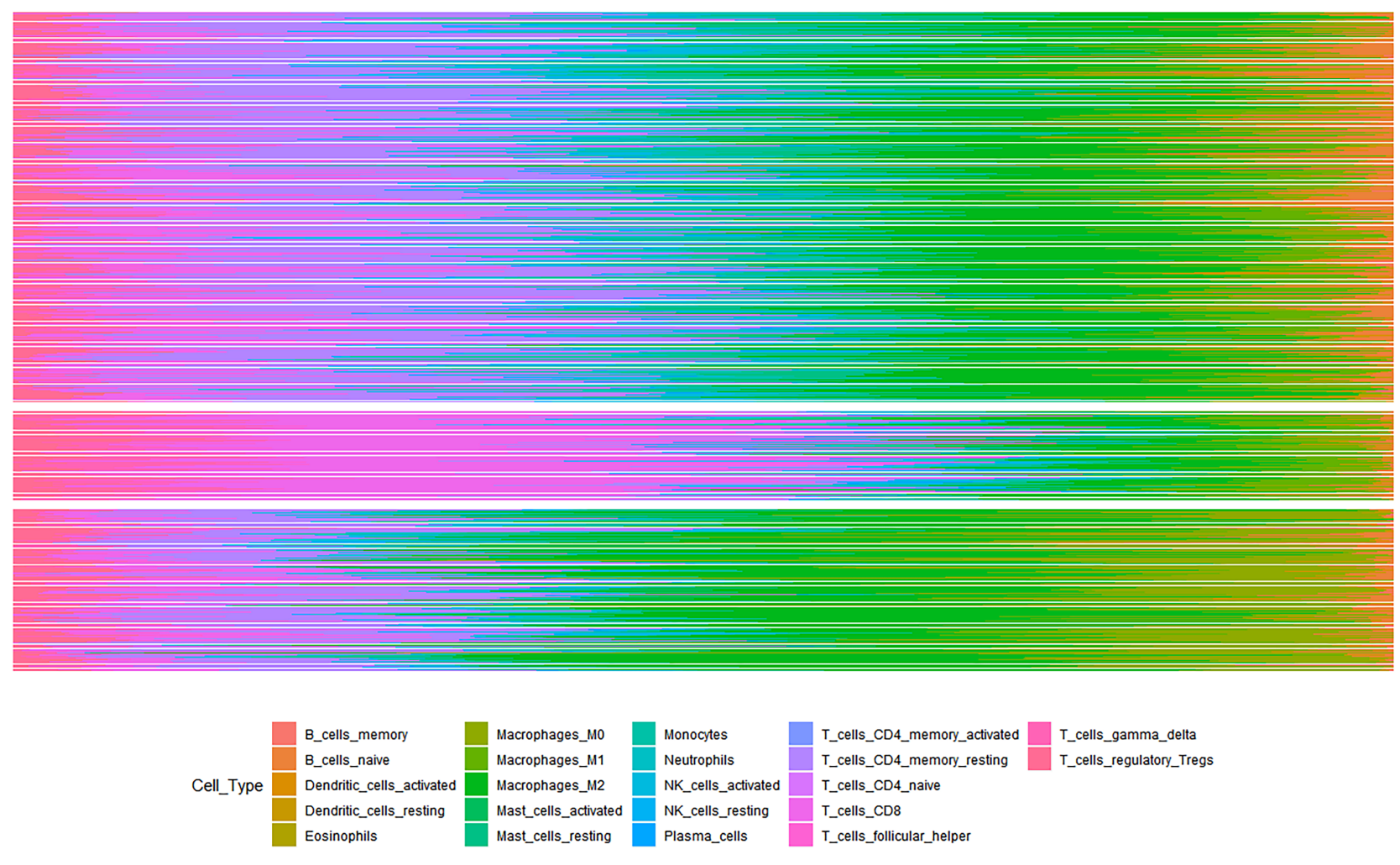

Figure 5

Immune clusters were associated with the prognosis of renal cancer patients. (A) Euclidean distance model identified three different immune clusters (cluster 1, cluster 2 and cluster 3). (B) Survival curves of 
cluster 1, 2 and 3 . Cluster 1 presented superior survival outcomes, while cluster 2 presented inferior survival outcomes $(p=0.0057)$. (C) The 3 clusters exhibited different compositions of 22 TIICs subsets.

\section{Supplementary Files}

This is a list of supplementary files associated with this preprint. Click to download.

- TableS1.xIsx

- FigS3.tif

- Figs2.tif

- Figs1.tif 